\title{
EFFECT OF A FULL PILATES GROUP EXERCISE PROGRAM ON TRANSVERSUS ABDOMINIS THICKNESS, DAILY FUNCTION AND PAIN IN WOMEN WITH CHRONIC LOW BACK PAIN
}

\author{
Noelia Goldberg' ${ }^{1}$, Asaf Weisman ${ }^{1}$, Snježana Schuster ${ }^{2}$, Gali Dar ${ }^{3}$, and Youssef Masharawi ${ }^{1}$ \\ ${ }^{1}$ Spinal Research Laboratory, Department of Physical Therapy, Stanley Steyer School of Health \\ Professions, Sackler Faculty of Medicine, Tel Aviv University, Israel \\ ${ }^{2}$ University of Applied Health Sciences, Zagreb, Croatia \\ ${ }^{3}$ University of Haifa, Department of Physical Therapy, Haifa, Israel
}

Original scientific paper

DOI $10.26582 / \mathrm{k} .53 .2 .15$

\begin{abstract}
:
The Pilates method is often used in the treatment of nonspecific low back pain (NSCLBP). The effect of Pilates group exercising (mat and apparatus combined) on women with NSCLBP is unknown. Studies on the Pilates method often use ultrasound (US) to measure transversus abdominis (TrA) thickness in one anatomical location. We recruited 40 women, 22 with NSCLBP and 18 healthy women (active control group), to join five weeks of biweekly Pilates group exercising (apparatus and mat). Measurements included TrA thickness (left and right) at rest, during active draw-in maneuver (ADIM), and its TrA\% change in three anatomical areas; Roland-Morris disability questionnaire (RMDQ); and pain level (VAS). Before the study, no asymmetry in TrA thickness was noted as well as no between-group differences in TrA-thickness in three anatomic areas (in rest and ADIM). Following the intervention, TrA thickness increased during rest in the healthy group in two areas. No differences were noted between the two groups in ADIM in the anatomical locations. No between-group differences were found before the intervention in the $\operatorname{Tr} \mathrm{A} \%$ change. Following the intervention, an increase in the TrA-\% change was noticed in the NSCLBP group. A decrease in the healthy group was observed in area C. No effects were noted on the RMDQ and VAS. Five weeks of Pilates group exercising increased TrA thickness in healthy women and its percentage changed in women with NSCLBP. Measuring TrA thickness with US should be performed in three different anatomical locations on both sides. This measuring procedure has good inter- and intra-rater reliability and the potential to reveal physiological effects.
\end{abstract}

Key words: group exercising, gender-specific medicine, backache

\section{Introduction}

Low back pain is a significant public health concern with a reported lifetime prevalence of $84 \%$ and a higher overall mean prevalence in women (Balagué, Mannion, Pellisé, \& Cedraschi, 2012; Hoy, et al., 2012). Often, low back pain persists with no identifiable pathology and has been labeled as nonspecific chronic low back pain (NSCLBP). Exercising appears to be the best available intervention for NSCLBP (Lizier, Perez, \& Sakata, 2012). Nonetheless, conflicting evidence exists about the effectiveness of exercise therapy's effect on NSCLBP (Lizier, et al., 2012). When considering group exercising versus individual treatments of patients with NSCLBP, group exercising is economically feasible for healthcare providers as it allows them to reach more clients with fewer therapists. It has also been shown that patients gain greater self-confidence (Lewis, et al., 2005). Weight-bearing (Beladev \& Masharawi, 2011) and non-weight-bearing (Masharawi \& Nadaf, 2013) group exercising was found to be effective in relation to the lumbar range of motion, pain levels, and function in women with NSCLBP. However, rotation exercising was found to be ineffective on the same parameters and population (Segal-Snir, Lubetzky, \& Masharawi, 2016). Further investigation is required regarding specific exercises that should be performed to decrease pain and improve function in patients with NSCLBP, particularly in the female population.

The Pilates method of exercising is often employed as a treatment for NSCLBP, yet its effec- 
tiveness has not been demonstrated to be superior to other forms of exercise (Kofotolis, Kellis, Vlachopoulos, Gouitas, \& Theodorakis, 2016; Yamato, et al., 2016). This method aims to activate the deep trunk muscles, mainly the transversus abdominis ( $\operatorname{Tr} A)$, which has been presumed to be related to LBP (Cruz-Díaz, Bergamin, Gobbo, MartínezAmat, \& Hita-Contreras, 2017). Thus far, most Pilates studies have compared the Pilates method with other forms of exercising (Lin, et al., 2016). So far, combined Pilates group exercising (mat and apparatus) effects on TrA morphology and daily functions in individuals with NSCLBP was not examined. The impact of various Pilates treatment protocols on TrA thickness is frequently assessed by ultrasound (US) imaging. Nevertheless, to date, TrA thickness has always been measured in only one anatomic location, even though TrA morphology changes along the muscle trajectory due to different fascicle orientations, length, and width (Urquhart, Barker, Hodges, Story, \& Briggs, 2005).

The importance of gender-specific medicine is a topic of ongoing debate (Ruiz \& Verbrugge, 1997), and the establishment of the International Society of Gender Medicine in 2012 helped increase awareness of the importance of the subject significantly (Shimokawa, 2015). The main principle of genderspecific medicine is that women exhibit inherently different biological traits than men and thus require other medical care (Ruiz \& Verbrugge, 1997). Also, clinical trials conducted on a mixed population of men and women present bias and cannot be automatically generalized exclusively to the female population (Bennett, 1993). As mentioned above, the prevalence of low back pain is higher in women than in men, and therefore this population requires special attention in scientific studies (Hoy, et al., 2012).

This study's primary aim was to examine the effect of combined Pilates group exercising (apparatus and mat) on the TrA thickness, daily function, and pain levels in women with NSCLBP compared with healthy women. The secondary aim was to test a novel US measuring procedure of TrA thickness in three anatomical locations.

\section{Methods}

The Institutional Review Boards of the authors' affiliated institutions approved this study. The inclusion criteria for the NSCLBP group included adult women (21-65 years of age) who were suffering from nonspecific central LBP (Balagué, et al., 2012) for $>12$ weeks. The exclusion criteria were: pregnancy, BMI $>30$, spine or abdominal operations, trauma, spondyloarthropathies, neurological symptoms in the limbs, neurological diseases, any ongoing physical exercise program, any previous treatments or medication during the last three months, and any experience with the Pilates method. Following an explanation of the study's nature, all volunteers signed a consent form and filled out a general health questionnaire to establish their capability to participate in the study. Out of 98 potential participants, 49 were eventually included in the study. Of the 49 participants, 26 met the participation criteria with NSCLBP, whereas 23 healthy subjects were assigned to an active comparison group. Before any testing, we obtained informed consent to protect the participants' rights according to the international good clinical practice rules.

All potential participants were screened by a single examiner who reviewed their general health statements and liaised with them only once at the beginning of the study. Consequently, this examiner allocated them into two groups: the NSCLBP group and the active healthy group. Following the assignment to the groups, participants were assessed by a different examiner blinded to their alloction, a physical therapist with 12 years of experience and trained in US imaging (examiner A - first author). This examiner collected the US outcome measures in two assessments: once before the intervention and then once more following the intervention period. Intra- and inter-rater reliability tests were performed on the first 10 participants by two therapists (A and $\mathrm{B}$ - fourth author), who had been trained in US imaging of the abdominal muscles. The intervention included biweekly 60-minute exercise sessions over five weeks (a total of 10 sessions). The exercises were selected from the classic Pilates repertoire, including working with Cadillac, Reformer, Wunda Chair, and mat. According to the classic Pilates method, the same routines were performed ten times in each session, in the same order. Every session began with 30 minutes of 16 mat exercises followed by 30 minutes of apparatus exercises in the following order (Figures 1 and 2): ten exercises on the Reformer (approx. 15 minutes), two exercises on the Cadillac (approx. 5 minutes), two exercises on the Wunda Chair, and two exercises on the Ladder Barrel (approx. 5 minutes). Two well-trained Pilates instructors with five years of experience and qualifications from the Pilates Method Alliance conducted all group sessions. All of the exercises employed in the study are thoroughly described in the supplementary material.

We measured TrA cross-sections in three different locations on both sides (right and left) using the Mindray $6600 \mathrm{~B}$-mode US unit with a convex $6 \mathrm{MHz}$ transducer. The participants were examined in a hook-lying position. The examiner then placed the transducer between the $12^{\text {th }}$ rib and the iliac crest's anterior aspect so that the hyperechoic line between the TrA and the thoracolumbar fascia was positioned at the right end of the image (Figure 3). Before the examination, the ADIM was described to the patient according to existing protocols (Richardson, et al., 2002; Teyhen, et al., 2005). 

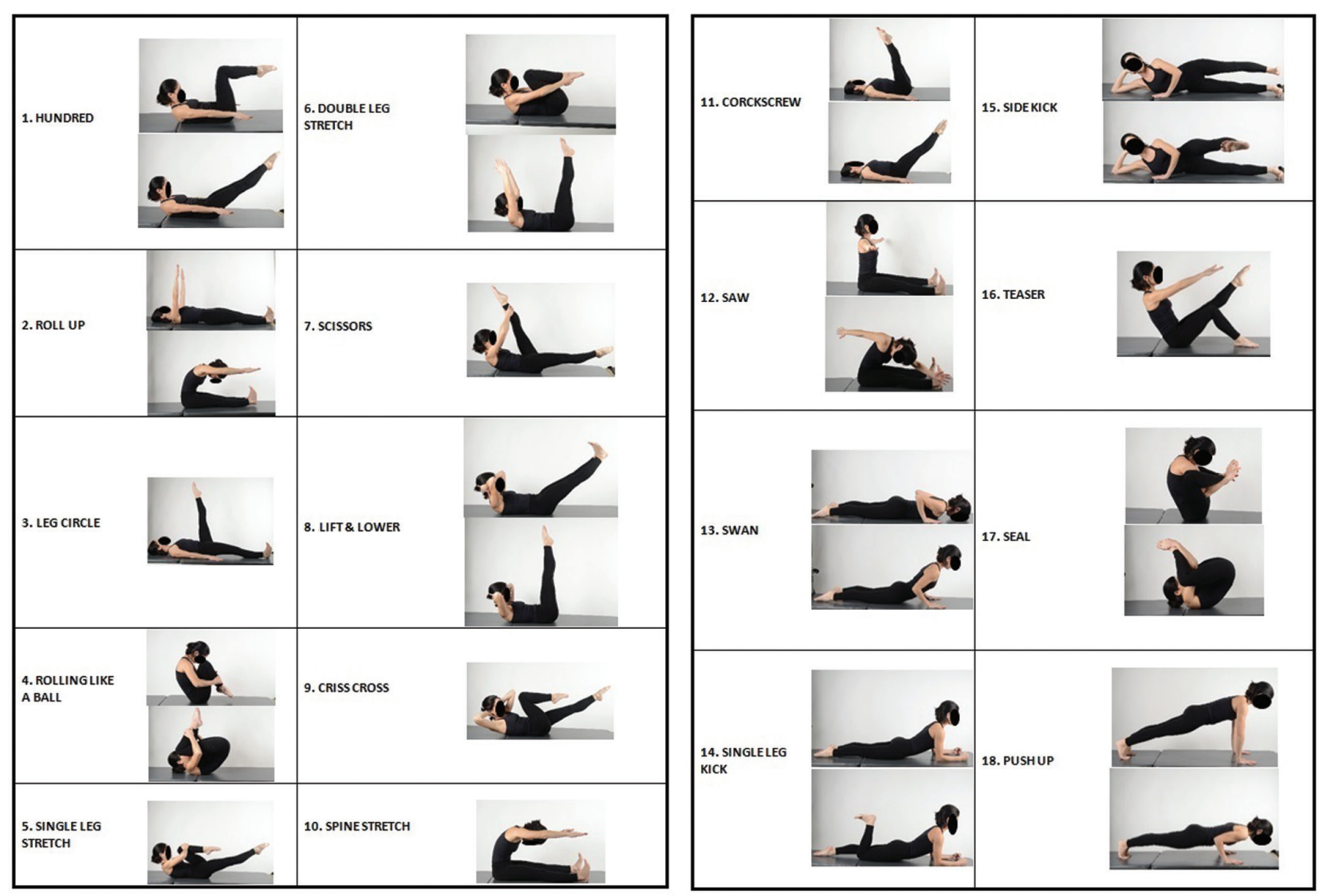

Figure 1. The protocol of Pilates mattress exercises.
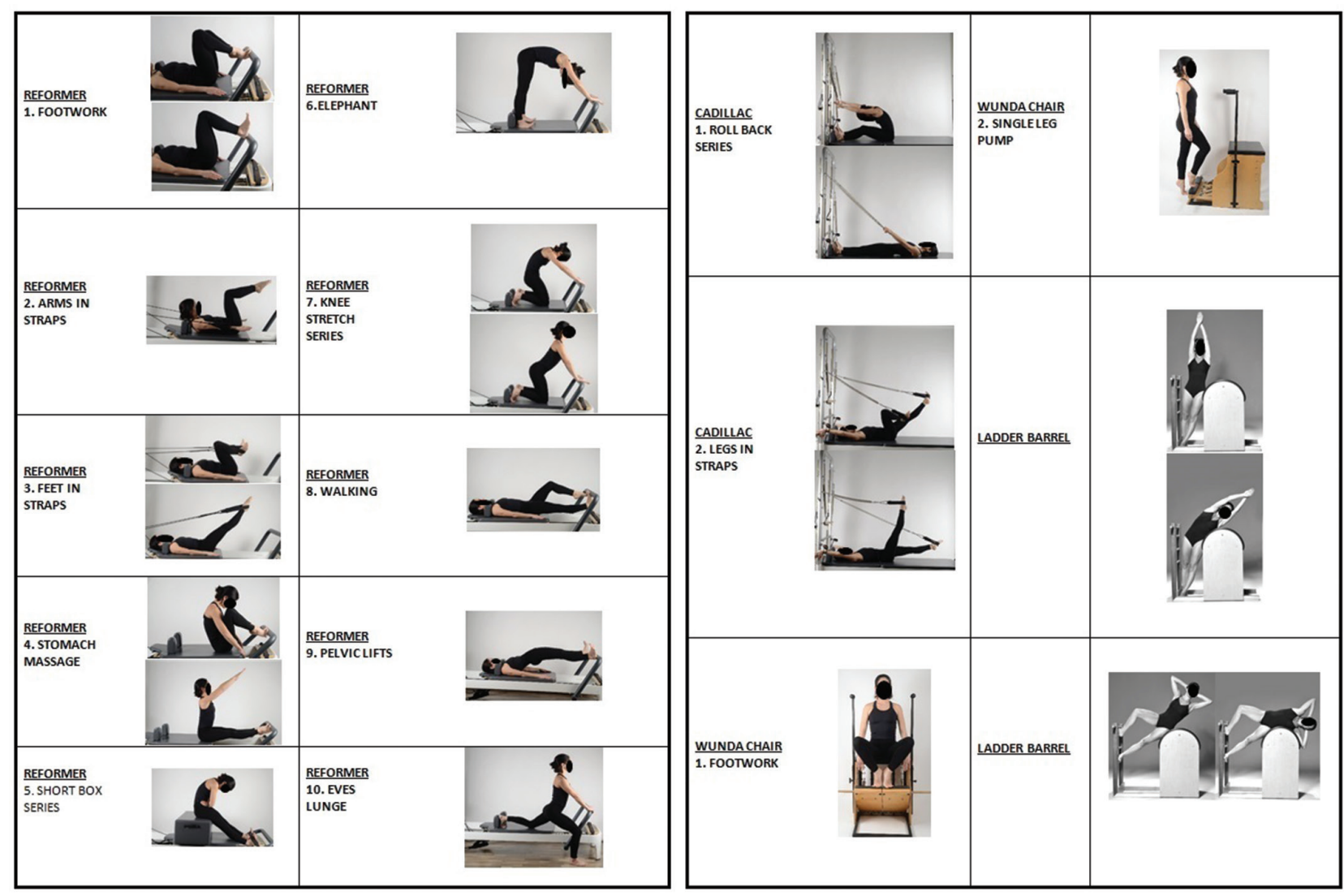

Figure 2. The protocol of Pilates apparatus exercises. 


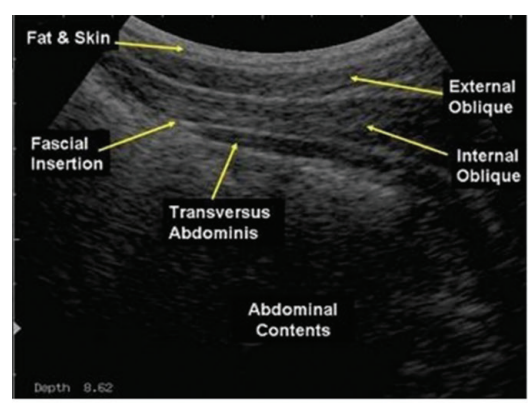

I

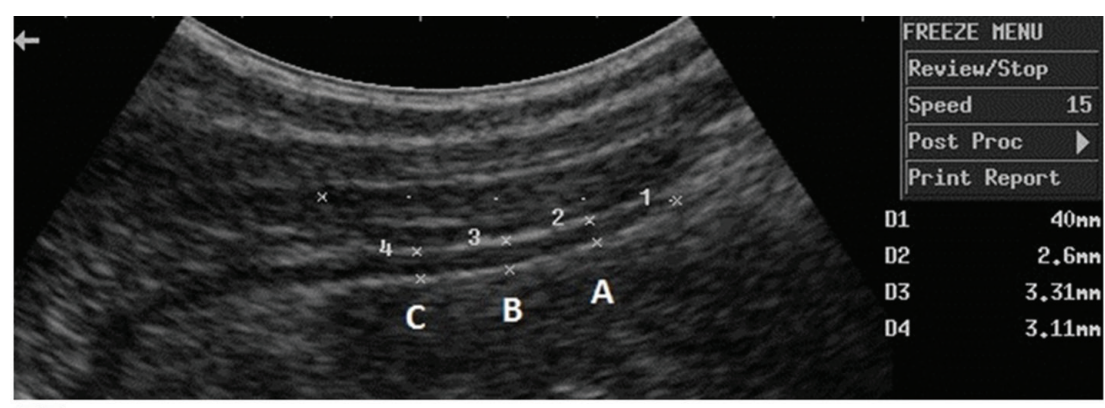

II

Figure 3. I-Ultrasound image of transversus abdominis (TrA); II-measurement of TrA at three different locations: A-at $10 \mathrm{~mm}$ from the fascial insertion, $B$-at $20 \mathrm{~mm}$ from the fascial insertion, and $C$-at $30 \mathrm{~mm}$ from the fascial insertion.

The protocol was practiced once as recommended. Following a 5-minute break, testing resumed. To control the participants' breathing, ADIM was executed during exhalation, and the image was acquired at the end of the normal exhalation. Three different locations were measured across the muscle from medial to lateral on both sides: Location $\mathrm{A}$ at $10 \mathrm{~mm}$ from the fascial insertion, location $\mathrm{B}$ at 20 $\mathrm{mm}$ from the fascial insertion, and location $\mathrm{C}$ at 30 $\mathrm{mm}$ from the fascial insertion (Figure 3).

TrA muscle thickness values while contracted were normalized based on the resting-thickness values described previously (Teyhen, et al., 2009). Accordingly, we calculated TrA percent of change to assess the relative changes in muscle thickness using the following formula:

\section{$\{(\operatorname{Tr} A$ active $-\operatorname{Tr}$ A rest $) / \operatorname{Tr} A$ rest $\times 100\}$}

(Teyhen, et al., 2009). We included the Visual Analogue Scale (VAS) and the Roland-Morris questionnaire (RMDQ) in assessing the NSCLBP group before and after the Pilates intervention.

Data were analyzed by an independent statistician using the Statistical Package for the Social Sciences, Version 23 (SPSS Inc., Chicago, IL, USA). Results of the measurements of each test were analyzed for mean, standard deviation (SD), and range. The Kolmogorov-Smirnov test verified the normal distribution of the variables (Lilliefors, 1967). The Student's $t$-tests were performed to establish differences between the groups before and after the intervention, and paired $t$-tests demonstrated any differences between the means, averages, and standard deviations of the continuous data. Analysis of variance (ANOVA) with repeated measures was performed on all symmetry dependent variables and was used to establish whether there were any differences between the tests. The inter-intraclass correlations (ICC) were calculated with $95 \%$ confidence intervals (CI). We considered a mean cutoff of ICC $>.75$ a priori as good. The Pearson's r correlation calculated correlations between parametric variables. The sample size was calculated using a desired minimal difference of $1 \mathrm{~mm}$. For a power of $80 \%$, alpha size of .05 , and an estimated $10 \%$ drop-out, the sample size recommended for each group was 20 .

\section{Results}

Nine subjects dropped out from the study before any exercise intervention due to pregnancy $(\mathrm{n}=2)$, fertility treatments $(\mathrm{n}=1)$, elective abdominal surgery $(n=1)$, dizziness $(n=1)$, and other personal reasons $(n=4)$. An intention-to-treat analysis was not performed, as all nine drop-outs occurred before the beginning of the intervention. Therefore, the final data analysis was based on 22 participants with NSCLBP and 18 healthy participants (Table 1). No significant differences were found between the groups' mean baseline $\left(\mathrm{t}_{0}\right)$ measures on all measured parameters (age, height, weight, and BMI) (.134 $<\mathrm{p}<$.810) (Table 1). Intra-examiner and inter-examiner reliability of all TrA thickness measures were found to be good $(.767<$ mean-ICC $<.772$, mean-CI $=95 \%$ ). All measured variables at all stages were normally distributed in the two groups (Kolmogorov-Smirnov: $.447<p<1.077$ ). Duration of pain was $8 \pm 7.4$ years. No significant differences were found in the RMDQ score (from $4.54 \pm 3.9$ to $3.95 \pm 3.86$, $\mathrm{p}=.337$ ) or in the VAS scale (from $3.64 \pm 2.42$ to $3.1 \pm 2.18, \mathrm{p}=.216$ ) before and after the intervention in the NSCLBP group. No correlations were found

Table 1. Subjects characteristics

\begin{tabular}{llcc}
\hline Variable & Group & Mean & Range \\
\hline \multirow{2}{*}{ Age (year) } & Healthy & $41.63( \pm 13.46)$ & $23.3-61.6$ \\
& NSCLBP & $38.86( \pm 11.36)$ & $23.4-58.9$ \\
Height (cm) & Healthy & $1.63( \pm 0.074)$ & $151-183$ \\
& NSCLBP & $1.64( \pm 0.048)$ & $157-173$ \\
& Healthy & $69.65( \pm 14.84)$ & $51-100$ \\
Weight $\mathbf{( k g )}$ & NSCLBP & $64.42( \pm 12.9)$ & $52-116$ \\
& Healthy & $25.91( \pm 5.36)$ & $20-36$ \\
BMI (kg/m²) & NSCLBP & $23.82( \pm 4.3)$ & $19-39$ \\
\hline
\end{tabular}


Table 2. Means of transversus abdominis muscle thickness and percentage of change in rest and ADIM

\begin{tabular}{|c|c|c|c|c|}
\hline \multicolumn{5}{|c|}{ Pre intervention $\left(t_{0}\right)$} \\
\hline Groups & & A & B & C \\
\hline \multirow{3}{*}{$\begin{array}{l}\text { Healthy } \\
n=23\end{array}$} & Rest (mm) & $3.27( \pm 0.75)$ & $3.54( \pm 0.76)$ & $3.47( \pm 0.77) £$ \\
\hline & ADIM (mm) & $4.84( \pm 1.02)$ & $5.62( \pm 1.22)$ & $5.63( \pm 1.1)$ \\
\hline & $\%$ change & $50.13( \pm 24.23)$ & $60.48( \pm 24.26)$ & $64.83( \pm 23.73)^{*}$ \\
\hline \multirow{3}{*}{$\begin{array}{l}\text { NSCLBP } \\
n=26\end{array}$} & $\operatorname{Rest}(\mathrm{mm})$ & $3.04( \pm 0.74)$ & $3.27( \pm 0.78)$ & $3.31( \pm 0.75)$ \\
\hline & ADIM (mm) & $4.45( \pm 1.15)$ & $5.18( \pm 1.19)$ & $5.37( \pm 1.22)$ \\
\hline & $\%$ change & $48.58( \pm 30.02)$ & $60.49( \pm 25.16)$ & $64.35( \pm 26.6) \dagger$ \\
\hline \multicolumn{5}{|c|}{ Post intervention $\left(t_{1}\right)$} \\
\hline Groups & & A & B & C \\
\hline \multirow{3}{*}{$\begin{array}{l}\text { Healthy } \\
n=18\end{array}$} & Rest (mm) & $3.43( \pm 0.68)$ & $3.68( \pm 0.6)$ & $3.86( \pm 0.79) £$ \\
\hline & ADIM (mm) & $5.44( \pm 1.23)$ & $5.95( \pm 1.24)$ & $5.92( \pm 1.16)$ \\
\hline & $\%$ change & $59.37( \pm 24.6)$ & $61.62( \pm 21.13)$ & $55.4( \pm 23.64)^{*}$ \\
\hline \multirow{3}{*}{$\begin{array}{l}\text { NSCLBP } \\
n=22\end{array}$} & Rest (mm) & $3.21( \pm 0.68)$ & $3.25( \pm 0.64)$ & $3.29( \pm 0.69)$ \\
\hline & ADIM (mm) & $4.92( \pm 1.27)$ & $5.51( \pm 1.35)$ & $5.75( \pm 1.29)$ \\
\hline & $\%$ change & $54.24( \pm 33.52)$ & $70.23( \pm 32.74)$ & $75.73( \pm 28.62) \dagger$ \\
\hline
\end{tabular}

Note. $£, \dagger,{ }^{*}-$ Significance within the same variable
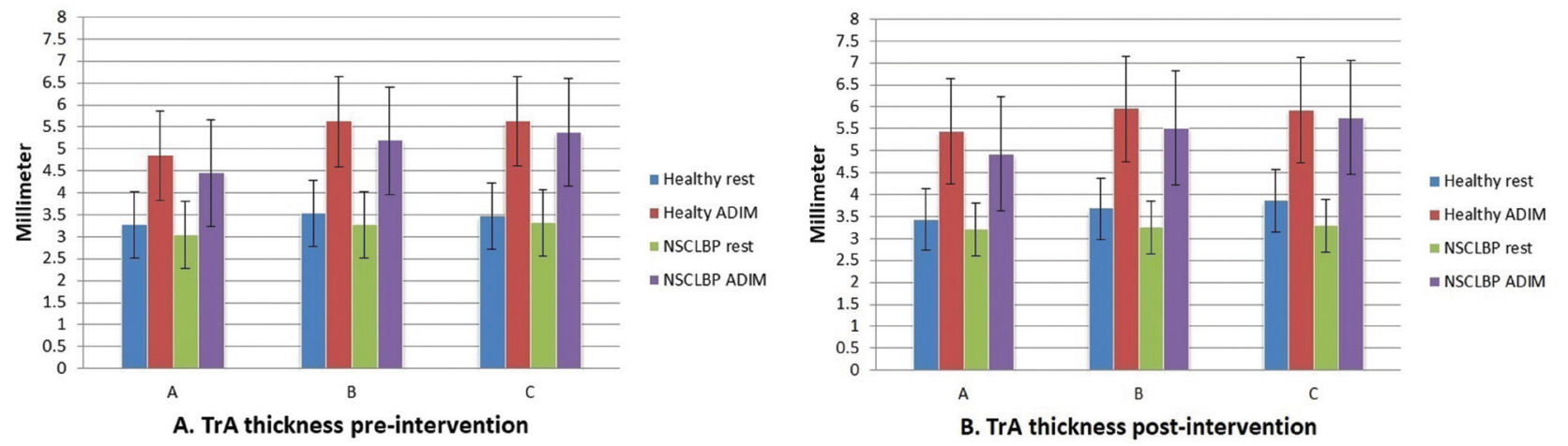

Figure 4. Transversus abdominis thickness pre-and post-intervention.

between any of the variables of both the primary (TrA thickness) and secondary outcome measures (VAS and RMDQ) ( $\mathrm{p}>05$ ).

- No significant left-right asymmetry in TrA thickness was found in either group in all measurements at rest $(.23<\mathrm{p}<.29)$ and during ADIM $(.21<\mathrm{p}<.43)$ (Table 2). Hence, the data of $\operatorname{TrA}$ thickness of both sides were combined to create a larger sample (NSCLBP group: $\mathrm{n}=22 \times 2=44$, healthy group: $n=18 \times 2=36$ ).

- Prior to the intervention, no significant differences were noted between the two groups in TrA thickness as well as in its percent of changes between rest and ADIM in all three anatomical locations (A, B, C) at rest $(.28<\mathrm{p}<.46)$ and during ADIM $(.23<\mathrm{p}<.44)$ (Table 2 and Figure 4A).

- Following the intervention, TrA thickness at rest was significantly greater only in the healthy group and only in anatomical locations $\mathrm{B}(\Delta=.43$ $\mathrm{mm})$ and $\mathrm{C}(\Delta=.57 \mathrm{~mm})(\mathrm{p}<.05)$. There were no significant differences in ADIM between the two groups in any of the three anatomical locations (Table 2 and Figure 4B). A significant increase was observed following the intervention in $\operatorname{Tr} A$ thickness percent of change (from $64.35 \% \pm 26.6$ to $75.73 \% \pm 28.62, \mathrm{p}<.05)$ in the NSCLBP group and a significant decrease in the healthy group (from $64.83 \% \pm 23.73$ to $55.4 \% \pm 23.64, \mathrm{p}<.05$ ) only in location C (Table 2).

\section{Discussion and conclusions}

Pilates in the community is often exercised in groups that combine two forms of exercises (mat and apparatus), and to the best of our knowledge, those aspects have never been examined. Furthermore, TrA thickness in the current study was measured in three anatomical locations on each side compared with one anatomical location reviewed in previous studies (Critchley, Pierson, \& Battersby, 2011; Cruz-Díaz, et al., 2017). The present results indicate that employing a full Pilates protocol for five weeks increased TrA thickness only in healthy 
women in two anatomical locations (B and C). The reason why changes in $\operatorname{Tr} A$ thickness following Pilates exercising acted differently in the three anatomical locations probably lies in the fact that TrA morphology varies along its trajectory with different fascicle orientation, length, and width (Urquhart, et al., 2005). Consequently, this intricate morphological structure of the TrA may provoke different responses of various anatomical parts to specific exercises (Urquhart, et al., 2005). A single cross-sectional location may fail to capture the full muscle volume; thus, multiple anatomical locations can better estimate muscle volume. Although the evidence and theoretical basis for measuring $\operatorname{Tr} \mathrm{A}$ thickness at various anatomical points conducted in the current study are preliminary, we suggest including these three anatomical locations in future studies on TrA thickness (Urquhart, et al., 2005).

The improvement of TrA thickness in the healthy participants was probably due to normal activation of the muscles during exercises as "pain inhibition" in the NSCLBP may have compromised normal activation (Freeman, Woodham, \& Woodham, 2010; Ruhe, Fejer, \& Walker, 2011). A longer period of exercising might have gradually overcome this inhibition. The current results support this as the percent of change in TrA thickness increased in the participants with NSCLBP even though no improvement was noticed in the muscle absolute thickness. The fact that TrA thickness had increased in the healthy participants may explain the reduction in the percent of change following the intervention.

In the participants with NSCLBP, no benefit occurred during the five-week mixed Pilates group protocol regarding pain levels and daily function. This finding is similar to a previous study with a six-week Pilates exercise regime that reported no pain intensity improvement (da Luz, et al., 2014). A longer duration of the Pilates exercise regime might benefit patients with chronic LBP with regard to pain intensity. Other studies with exercise regimes lasting longer than eight weeks reported favorable pain intensity outcomes (Cruz-Díaz, et al., 2017; Lee, Hyun, \& Kim, 2014). Similarly, previous studies with longer periods of interventions demonstrated better outcomes from the Pilates exercise protocol in terms of function than other minimal interventions (Cruz-Díaz, et al., 2017; Patti, et al., 2015).

Our five-week protocol initiated TrA thickness improvement, yet it is possible that the intervention period was not long enough for improving pain intensity or daily functions in women with NSCLBP. This detail contrasts the reduction in disability and improvement in daily function presented by a recent study on Pilates, where mat exercises were compared with apparatus exercises (Cruz-Díaz, et al., 2017). The fact that no functional improvement was observed in the current study weakens their explanation of a possible placebo effect on participants with NSCLBP. It is noteworthy that the participants with NSCLBP in the present study had relatively low scores of functional disability, to begin with, and were able to attend Pilates in the community. Thus it would not be realistic to expect further functional improvement. Finally, other factors may also be responsible for different functional outcomes, including patients' enthusiasm, study population, and instructor competence.

Previous studies on healthy participants have demonstrated abdominal muscle left-right asymmetry at rest (Rankin, Stokes, \& Newham, 2006; Teyhen, et al., 2012). In the current study, no significant asymmetry in TrA thickness was observed during rest or ADIM in either group. These findings should be taken into account when considering whether morphological changes of abdominal musculature are predictors or indicators of LBP and clinical improvement as was previously suggested (Ghamkhar, Emami, Mohseni-Bandpei, \& Behtash, 2011; Kiesel, Underwood, Mattacola, Nitz, \& Malone, 2007). According to our results, the clinical utility of investigating possible asymmetry in the TrA is not very high in determining diagnostic or treatment assumptions or as a prognostic parameter in women with NSCLBP. The fact that no asymmetry was observed in either of three anatomical locations in both groups at rest and during ADIM, should strongly emphasize the conclusion that $\operatorname{TrA}$ asymmetry should no longer be considered in any future relevant studies. There is no clinical utility in measuring TrA thickness during ADIM compared with rest, as no TrA thickness changes were observed during this procedure.

Finally, this study's primary purpose was to examine Pilates' effect on TrA thickness in healthy women and women with NSCLBP. Thus, it was necessary to use two active groups rather than just comparing the NSCLBP group to a non-active NSCLBP control group. This detail and a wide age range of the participants (21-65) may have limited our clinical conclusion, yet it strengthened our conclusion regarding TrA thickness. Lastly, the current study is the first to provide gender-specific evidence for the influences of a short-term Pilates program on TrA thickness in healthy women and women with NSCLBP.

Five weeks of the combined Pilates group exercising increased the TrA thickness in healthy women and the muscle percentage of change in women with NSCLBP, yet did not moderate functional disability and VAS in women with NSCLBP. Future studies on the Pilates method's effect on TrA should perform US measurements in three different anatomical locations (from medial to lateral and at 10,20 , and $30 \mathrm{~mm}$ from the fascial insertion). This method has been shown to have good intra- and inter-rater reliability. 


\section{References}

Balagué, F., Mannion, A.F., Pellisé, F., \& Cedraschi, C. (2012). Non-specific low back pain. The Lancet, 379(9814), 482-491. doi: 10.1016/S0140-6736(11)60610-7

Beladev, N., \& Masharawi, Y. (2011). The effect of group-exercising on females with nonspecific chronic low back pain in a sitting position: A pilot study. Journal of Back and Musculoskeletal Rehabilitation, 24(3), 181-188. doi: 10.3233/BMR-2011-0292

Bennett, J.C. (1993). Inclusion of women in clinical trials-Policies for population subgroups. New England Journal of Medicine, 329(4), 288-292. doi: 10.1056/NEJM199307223290428

Critchley, D.J., Pierson, Z., \& Battersby, G. (2011). Effect of pilates mat exercises and conventional exercise programmes on transversus abdominis and obliquus internus abdominis activity: Pilot randomised trial. Manual Therpy, 16(2), 183-189. doi: 10.1016/j.math.2010.10.007

Cruz-Díaz, D., Bergamin, M., Gobbo, S., Martínez-Amat, A., \& Hita-Contreras, F. (2017). Comparative effects of 12 weeks of equipment based and mat Pilates in patients with chronic low back pain on pain, function and transversus abdominis activation. A randomized controlled trial. Complementary Therapies in Medicine, 33, 72-77. doi: 10.1016/j.ctim.2017.06.004

da Luz, J.M.A., Costa, L.O.P., Fuhro, F.F., Manzoni, A.C.T., Oliveira, N.T.B., \& Cabral, C.M.N. (2014). Effectiveness of mat pilates or equipment-based pilates exercises in patients with chronic nonspecific low back pain: A randomized controlled trial. Physical Therapy, 94(5), 623-631. doi: 10.2522/ptj.20130277

Freeman, M.D., Woodham, M.A., \& Woodham, A.W. (2010). The role of the lumbar multifidus in chronic low back pain: A review. Physical Medicine and Rehabilitation, 2(2), 142-146. doi: 10.1016/j.pmrj.2009.11.006

Ghamkhar, L., Emami, M., Mohseni-Bandpei, M.A., \& Behtash, H. (2011). Application of rehabilitative ultrasound in the assessment of low back pain: A literature review. Journal of Bodywork and Movement Therapies, 15(4), 465-477. doi: 10.1016/j.jbmt.2010.07.003

Hoy, D., Bain, C., Williams, G., March, L., Brooks, P., Blyth, F., . ., \& Buchbinder, R. (2012). A systematic review of the global prevalence of low back pain. Arthritis and Rheumatology, 64(6), 2028-2037. doi: 10.1002/art.34347

Kiesel, K.B., Underwood, F.B., Mattacola, C.G., Nitz, A.J., \& Malone, T.R. (2007). A comparison of select trunk muscle thickness change between subjects with low back pain classified in the treatment-based classification system and asymptomatic controls. Journal of Orthopedic Sports Physiscal Therapy, 37(10), 596-607. doi: 10.1002/art.34347

Kofotolis, N., Kellis, E., Vlachopoulos, S.P., Gouitas, I., \& Theodorakis, Y. (2016). Effects of Pilates and trunk strengthening exercises on health-related quality of life in women with chronic low back pain. Journal of Back and Musculoskeletal Rehabilitation, 29(4), 649-659. doi: 10.3233/BMR-160665

Lee, C.-W., Hyun, J., \& Kim, S.G. (2014). Influence of pilates mat and apparatus exercises on pain and balance of businesswomen with chronic low back pain. Journal of Physical Therapy Science, 26(4), 475-477. doi: 10.1589/ jpts. 26.475

Lewis, J.S., Hewitt, J.S., Billington, L., Cole, S., Byng, J., \& Karayiannis, S. (2005). A randomized clinical trial comparing two physiotherapy interventions for chronic low back pain. Spine, 30(7), 711-721. doi: 10.1097/01. brs.0000157469.27779.de

Lilliefors, H.W. (1967). On the Kolmogorov-Smirnov test for normality with mean and variance unknown. Journal of American Statistics Association, 62(318), 399-402.

Lin, H.-T., Hung, W.-C., Hung, J.-L., Wu, P.-S., Liaw, L.-J., \& Chang, J.-H. (2016). Effects of Pilates on patients with chronic nonspecific low back pain: A systematic review. Journal of Physical Therapy Science, 28(10), 29612969. doi: $10.1589 /$ jpts.28.2961

Lizier, D.T., Perez, M.V., \& Sakata, R.K. (2012). Exercises for treatment of nonspecific low back pain. Brazilan Journal of Anesthesiology, 62(6), 838-846. doi: 10.1016/S0034-7094(12)70183-6

Masharawi, Y., \& Nadaf, N. (2013). The effect of non-weight bearing group-exercising on females with nonspecific chronic low back pain: A randomized single blind controlled pilot study. Journal of Back and Musculoskelet Rehabilitation, 26(4), 353-359. doi: 10.3233/BMR-130391

Patti, A., Bianco, A., Paoli, A., Messina, G., Montalto, M.A., Bellafiore, M., . ., \& Palma, A. (2015). Effects of Pilates exercise programs in people with chronic low back pain: A systematic review. Medicine, 94(4). doi: 10.1097/ MD.0000000000000383

Rankin, G., Stokes, M., \& Newham, D.J. (2006). Abdominal muscle size and symmetry in normal subjects. Muscle and Nerve, 34(3), 320-326. doi: 10.1002/mus.20589

Richardson, C.A., Snijders, C.J., Hides, J.A., Damen, L., Pas, M.S., \& Storm, J. (2002). The relation between the transversus abdominis muscles, sacroiliac joint mechanics, and low back pain. Spine, 27(4), 399-405.

Ruhe, A., Fejer, R., \& Walker, B. (2011). Center of pressure excursion as a measure of balance performance in patients with nonspecific low back pain compared to healthy controls: A systematic review of the literature. European Spine Journal, 20(3), 358-368. doi: 10.1007/s00586-010-1543-2

Ruiz, M.T., \& Verbrugge, L.M. (1997). A two way view of gender bias in medicine. Journal of Epidemiology and Community Health, 51(2), 106. doi: 10.1136/jech.51.2.106 
Segal-Snir, Y., Lubetzky, V., \& Masharawi, Y. (2016). Rotation exercise classes did not improve function in women with nonspecific chronic low back pain: A randomized single blind controlled study. Journal of Back and Musculoskeletal Rehabilitation, 29(3), 467-475. doi: 10.3233/BMR-150642

Shimokawa, H. (2015). The history and perspective of gender-specific medicine in Japan. Nihon Rinsho, 73(4), 547-550.

Teyhen, D.S., Bluemle, L.N., Dolbeer, J.A., Baker, S.E., Molloy, J.M., Whittaker, J., \& Childs, J.D. (2009). Changes in lateral abdominal muscle thickness during the abdominal drawing-in maneuver in those with lumbopelvic pain. Journal of Orthopedic Sports Physical Therapy, 39(11), 791-798. doi: 10.2519/jospt.2009.3128

Teyhen, D.S., Childs, J.D., Stokes, M.J., Wright, A.C., Dugan, J.L., \& George, S.Z. (2012). Abdominal and lumbar multifidus muscle size and symmetry at rest and during contracted states. Journal of Ultrasound in Medicine, 31(7), 1099-1110. doi: 10.7863/jum.2012.31.7.1099

Teyhen, D.S., Miltenberger, C.E., Deiters, H.M., Del Toro, Y.M., Pulliam, J.N., Childs, J.D., ..., \& Flynn, T.W. (2005). The use of ultrasound imaging of the abdominal drawing-in maneuver in subjects with low back pain. Journal of Orthopedic Sports Physical Therapy, 35(6), 346-355. doi: 10.2519/jospt.2005.35.6.346

Urquhart, D.M., Barker, P.J., Hodges, P.W., Story, I.H., \& Briggs, C.A. (2005). Regional morphology of the transversus abdominis and obliquus internus and externus abdominis muscles. Clincal Biomechanics, 20(3), 233-241. doi: 10.1016/j.clinbiomech.2004.11.007

Yamato, T.P., Maher, C.G., Saragiotto, B.T., Hancock, M.J., Ostelo, R.W., Cabral, C., . . Costa, L.O. (2015). Pilates for low back pain. Sao Paulo Medical Journal, 134(4), 366-367. doi: 10.1002/14651858.CD010265.pub2

Submitted: March 23, 2021

Accepted: October 12, 2021

Published Online First: December 8, 2021

Correspondence to:

Assoc. Prof. Youssef Masharawi, Ph.D.

Spinal Research Laboratory,

Department of Physical Therapy

Stanley Steyer School of Health Professions,

Sackler Faculty of Medicine

Tel Aviv University, Israel

Phone: 00-972-50-7288737;

E-mail: yossefm@tauex.tau.ac.il 